\title{
Fast neutrino-flavor conversion in the preshock region of core-collapse supernovae
}

\author{
Taiki Morinaga $\odot,{ }^{1}$ Hiroki Nagakura, ${ }^{2}$ Chinami Kato $\odot,{ }^{3}$ and Shoichi Yamada ${ }^{1}$ \\ ${ }^{1}$ Graduate School of Advanced Science and Engineering, Waseda University, 3-4-1 Okubo, Shinjuku, Tokyo 169-8555, Japan \\ ${ }^{2}$ Department of Astrophysical Sciences, Princeton University, Princeton, New Jersey 08544, USA \\ ${ }^{3}$ Department of Aerospace Engineering, Tohoku University, 6-6-01 Aramaki-Aza-Aoba, Aoba-ku, Sendai 980-8579, Japan
}

(Received 28 September 2019; revised manuscript received 4 December 2019; accepted 24 January 2020; published 25 February 2020)

\begin{abstract}
We make a strong case that the fast neutrino-flavor conversion, one of the collective flavor oscillation modes, commonly occurs in core-collapse supernovae (CCSNs). It is confirmed in the numerical data obtained in realistic simulations of CCSNs, but the argument is much more generic and applicable universally: The coherent neutrinonucleus scattering makes the electron lepton number (ELN) change sign at some inward direction and trigger the flavor conversion in the outward direction in the preshock region. Although the ELN crossing is tiny and that is why it has eluded recognition so far, it is still large enough to induce the flavor conversion. Our findings may have important observational consequences for CCSN neutrinos.
\end{abstract}

DOI: 10.1103/PhysRevResearch.2.012046

\section{INTRODUCTION}

Neutrinos will give us vital clues not only to the explosion mechanism of core-collapse supernovae (CCSNs) but also to their flavor structures. In fact, the prediction of the luminosities and energy spectra for all neutrino species requires taking into account neutrino oscillations appropriately. This is more difficult than previously thought, however, if collective neutrino oscillations [1-21], which are the flavor oscillations induced by neutrinos themselves in dense neutrino gases, occur, since they are nonlinear phenomena described with integro-partial-differential equations. No consensus has been reached thus far on whether, when, and how the collective oscillation occurs in CCSNs. In this Rapid Communication we make a strong case that the fast neutrino-flavor conversion, one of the collective neutrino oscillation modes that can be self-sustained without a mass term, should commonly occur in the postbounce phase of CCSNs.

The fast flavor conversion has been extensively studied in the literature [22-36]. A convenient criterion for its occurrence is supposed to be the electron lepton number (ELN) crossing, i.e., the number of $v_{e}$ is dominant over that of $\bar{v}_{e}$ in some propagation directions, whereas $\bar{v}_{e}$ overwhelms $v_{e}$ in the other directions. Tamborra et al. [18] searched for such ELN crossings in the numerical data of CCSN simulations under the assumption of spherical symmetry. Paying attention mainly to outward-going neutrinos, they reported negative results. More recently, such investigations were extended to the results of multidimensional simulations [30,34]. Abbar et al. [30] found the ELN crossing in some extended domains

Published by the American Physical Society under the terms of the Creative Commons Attribution 4.0 International license. Further distribution of this work must maintain attribution to the author(s) and the published article's title, journal citation, and DOI. in the postshock region. On the other hand, Delfan Azari et al. [34] reported no detection of ELN crossing based on the two-dimensional (2D) CCSN model in [37]. We stress that these results depend strongly on multidimensional effects and may change from model to model.

In this Rapid Communication we discuss the possibility of the fast flavor conversion, based on a more robust argument. We focus on the preshock region. This is the region ahead of the shock wave, in which cold matter mainly composed of heavy nuclei is imploding toward the shock. We argue that the ELN crossing is produced rather commonly by the coherent scattering of neutrinos on these heavy nuclei, with $\bar{v}_{e}$ being scattered more often than $v_{e}$, which sets the stage for the fast flavor conversion.

Capozzi et al. [31] pointed out recently that collisional processes are important to generate the fast flavor conversion. What they had in mind in their paper, however, is completely different from what we consider in this Rapid Communication. They studied scatterings on nucleons that occur in the vicinity of the neutrinosphere whereas we investigate scatterings on nuclei in the region at much larger radii. Cherry et al. [8,12], Cirigliano et al. [19], and Zaizen et al. [21] also explored the possible effect of scatterings under the assumptions of spherical symmetry and stationarity. It is well known, however, that the flavor eigenstates tend to be stabilized under such restrictions $[11,15,16]$. These assumptions, as well as the crude approximations they employed, unfortunately obscured the role of the fast flavor conversion.

As we will see below, our argument is quite simple and robust: The existence of ELN crossing is demonstrated analytically; it is then vindicated by more realistic CCSN simulations. Note that our findings have been overlooked so far probably because the ELN crossing is tiny. However, such a minuscule crossing is actually large enough for the fast flavor conversion to grow substantially. It is also intriguing that the flavor conversion always occurs in the outward direction, which may hence have an impact on the terrestrial observation 
of supernova neutrinos. Note that since our findings are based on linear analysis, the subsequent nonlinear evolutions remain to be investigated to obtain the eventual neutrino signal, which is quite a tough problem.

\section{BACKWARD SCATTERING ON HEAVY NUCLEI}

The main claim of this paper is that coherent scatterings of neutrinos on heavy nuclei produce the ELN crossing in the preshock region, which is tiny but still sufficient to induce the fast flavor conversion. Interestingly, the conversion occurs only in the outward direction, i.e., it is classified as convective instability $[26,33,38-40]$. We will substantiate this contention shortly.

The shock wave generated at the core bounce is stalled in the core and becomes an accretion shock at $r \sim 200 \mathrm{~km}$. Matter outside this stagnant shock is cold and hence mainly composed of heavy nuclei and is falling almost freely onto the shock front. Neutrinos emitted from the neutrinosphere located much deeper inside $(r \lesssim 50 \mathrm{~km})$ are moving outward almost freely outside the shock, since the matter density is low there. A small fraction of these neutrinos are backscattered by nuclei, however, and produce the inward-going population. Since the scattering cross sections are proportional to the squared energies of neutrinos and $\bar{v}_{e}$ has higher energies than $v_{e}$ on average while the luminosities are similar between them, the inward-going population is dominated by $\bar{v}_{e}$.

This can be demonstrated more quantitatively with the so-called bulb model, in which neutrinos are emitted from the neutrino surface half-isotropically. For concreteness, we assume that the distribution function of the neutrino $f_{v}(E, \boldsymbol{v})$ is proportional to $E^{\alpha_{v}} e^{-\left(3+\alpha_{v}\right) E / \bar{E}_{v}}$, in which $E$ and $\boldsymbol{v}$ are, respectively, the neutrino energy and the unit vector to specify the flight direction [9,41-43]; the index $v$ represents $\nu_{e}$ or $\bar{\nu}_{e}$ hereafter; and $\alpha_{v}$ and $\bar{E}_{v}$ are parameters. Without interactions with matter, all neutrinos are going outward, being confined in a cone. Their angular distributions $\mathscr{G}_{v}(\boldsymbol{v}) \equiv$ $\sqrt{2} G_{F} \int_{0}^{\infty} \frac{d E E^{2}}{2 \pi^{2}} f_{v}(E, \boldsymbol{v})$ are given as [43]

$$
\begin{aligned}
\mathscr{G}_{v}^{\text {bulb }}(\mu)= & 2 \mathrm{~cm}^{-1}\left(\frac{50 \mathrm{~km}}{R_{v}}\right)^{2}\left(\frac{L_{v}}{10^{52} \mathrm{ergs} / \mathrm{s}}\right)\left(\frac{10 \mathrm{MeV}}{\bar{E}_{v}}\right) \\
& \times \Theta\left(\mu-\sqrt{1-\left(R_{v} / r\right)^{2}}\right)
\end{aligned}
$$

where $R_{v}$ is the radius of the neutrinosphere, $L_{v}$ and $\bar{E}_{v}$ are the luminosity and average energy of neutrino, respectively, $\mu$ is the cosine of the zenith angle measured from the local radial direction, and $\Theta$ is the step function. The ELN angular distribution is given by $\mathscr{G}_{v_{e}}-\mathscr{G}_{\bar{v}_{e}}$. In most of realistic simulations we find that the ELN is positive and its intensity is of the order of $\gtrsim 10^{-1} \mathrm{~cm}^{-1}$ in the outward direction $(\mu \sim 1)$.

The population of inward-going neutrinos $(\mu<0)$ can be estimated from this outward-going population and the matter distribution as follows. The density profile outside the shock front is approximately expressed as $\rho(r) \propto r^{-\beta}$ as a function of radius $r$. The rate of coherent scattering is estimated with the formula given in [44]. Then the angular distribution of $v$ is derived by line integrations of the rate of the backward scattering as [43]

$$
\begin{aligned}
\mathscr{G}_{v}^{\text {scat }}(\mu) \simeq & 2 \times 10^{-5} \mathrm{~cm}^{-1} \frac{4+\alpha_{v}}{\left(3+\alpha_{v}\right)(3+\beta)}\left(\frac{A}{56}\right) \\
& \times\left(\frac{\rho_{\mathrm{sh}}}{10^{7} \mathrm{~g} / \mathrm{cm}^{3}}\right)\left(\frac{R_{\mathrm{sh}}}{200 \mathrm{~km}}\right)^{\beta}\left(\frac{200 \mathrm{~km}}{r}\right)^{1+\beta} \\
& \times\left(\frac{L_{v}}{10^{52} \mathrm{ergs} / \mathrm{s}}\right)\left(\frac{\bar{E}_{v}}{10 \mathrm{MeV}}\right) \\
& \times\left[(\mu+1)+\frac{1}{4}\left(\frac{R_{v}}{r}\right)^{2}\right]
\end{aligned}
$$

up to the lowest order of $\mu+1$ and $R_{v} / r$, where $A$ is the average mass number at the scattering site and is assumed to be constant [43], $R_{\mathrm{sh}}$ is the shock radius, and $\rho_{\mathrm{sh}}$ is the matter density just outside the shock front. The leading angular dependence reflects the fact that the coherent scattering is strongly forward peaked, $R \propto 1+\cos \theta$, where $R$ and $\theta$ are the scattering kernel and the scattering angle, respectively [44]. In the limit of $r \rightarrow \infty$, the outward-going neutrinos become all radially going and there is no neutrino going radially inward. At finite radii, however, there remains a small finite contribution, giving the second term in the last factor. Note that the difference in $R_{v}$ between $v_{e}$ and $\bar{v}_{e}$ is included only in this term. As a result, the ELN ( $\left.\mathscr{G}_{v_{e}}^{\text {scat }}-\mathscr{G}_{\bar{v}_{e}}^{\text {scat }}\right)$ becomes negative as long as $L_{v} \bar{E}_{v}$ is larger for $\bar{v}_{e}$ than for $\nu_{e}$ at angles that satisfy $1 \gg 1+\mu \gg\left(R_{\nu} / r\right)^{2} / 4$. We find that the ratio $\epsilon \equiv L_{\bar{v}_{e}} \bar{E}_{\bar{v}_{e}} / L_{v_{e}} \bar{E}_{v_{e}}$ is indeed larger than unity in realistic situations at the late postbounce phase $t_{\mathrm{pb}} \gtrsim 50 \mathrm{~ms}$ (see Fig. 3). The absolute value of ELN is estimated typically to be $\gtrsim 10^{-6} \mathrm{~cm}^{-1}$, which will be also vindicated later by realistic simulations.

The different signs of ELN for the outward and inward directions imply that an ELN crossing occurs in between. The growth rate of the fast flavor conversion is roughly given by the geometric mean of the ELN intensities at their positive and negative parts [see Eq. (8)]. ${ }^{1}$ It is estimated to be $\gtrsim \sqrt{\left(10^{-1} \mathrm{~cm}^{-1}\right)\left(10^{-6} \mathrm{~cm}^{-1}\right)} \gtrsim 10^{-4} \mathrm{~cm}^{-1}=$ $1 /(100 \mathrm{~m})=1 /(0.3 \mu \mathrm{s})$, which means that the growth length and time are both much shorter than the typical scales of length $(\gtrsim 10 \mathrm{~km})$ and time $(\gtrsim 10 \mathrm{~ms})$ of variations in the background matter in CCSNs.

\section{GROWTH RATES OF FLAVOR CONVERSION}

Before moving to the realistic numerical models, we give here some mathematical formulas that will be employed there for quantitative analyses. The initial phase of the collective neutrino flavor conversion can be studied by the linear stability analysis [25]. Flavor evolutions are described by the kinetic equations for the density matrices of neutrinos $f$,

$$
v \cdot \partial \mathrm{f}(x, \Gamma)=-i[\mathrm{H}(x, \Gamma), \mathrm{f}(x, \Gamma)]+\mathcal{C}[\mathrm{f}],
$$

\footnotetext{
${ }^{1}$ For the so-called two-beam model, in which the ELN distribution is given by $\mathscr{G}(\boldsymbol{v})=4 \pi\left[\mathscr{G}_{1} \delta\left(\boldsymbol{v}-\boldsymbol{v}_{1}\right)+\mathscr{G}_{2} \delta\left(\boldsymbol{v}-\boldsymbol{v}_{2}\right)\right]$ with model constants $\mathscr{G}_{1}, \mathscr{G}_{2}, \boldsymbol{v}_{1}$ and $\boldsymbol{v}_{2}$, in fact, the growth rate is given by $\sigma=\left(1-\boldsymbol{v}_{1} \cdot \boldsymbol{v}_{2}\right) \operatorname{Re}\left(\sqrt{-\mathscr{G}_{1} \mathscr{G}_{2}}\right)$.
} 
where $x \equiv(t, \boldsymbol{x})$ defines the position in space-time, $\Gamma \equiv$ $(E, v)$ specifies the energy and the flight direction, and the flavor-isospin convention $\mathrm{f}(E)=\mathrm{f}_{v}(E)$ and $\mathrm{f}(-E)=-\mathrm{f}_{\bar{v}}(E)$ with $E>0$ is employed, with $\left(v^{\mu}\right) \equiv(1, v)$; the Hamiltonian $\mathrm{H}$ is given as $\mathrm{H}(x, \Gamma)=\mathrm{H}_{\mathrm{vac}}(E)+\mathrm{H}_{\text {int }}(x, v)$ with the vacuum-mass term $\mathrm{H}_{\mathrm{vac}}(E) \equiv \mathrm{M}^{2} / 2 E$ and the potential term $\mathrm{H}_{\text {int }}(x, \boldsymbol{v}) \equiv v \cdot \Lambda(x)$, in which $\mathrm{M}^{2}$ is the mass-squared matrix and $\Lambda$ is the 4-current of leptons defined as $\Lambda^{\mu}(x) \equiv$ $\sqrt{2} G_{F}\left[\operatorname{diag}\left[\left\{j_{\alpha}^{\mu}(x)\right\}\right]+\int d \Gamma \mathrm{f}(x, \Gamma) v^{\mu}\right]$, with $j^{\mu}$ the number current of the charged lepton specified by $\alpha$ and $\int d \Gamma \equiv$ $\int_{-\infty}^{\infty} \frac{d E E^{2}}{2 \pi^{2}} \int \frac{d^{2} v}{4 \pi} ; \mathcal{C}$ is the collision term.

In the region of our current concern, $H_{\mathrm{vac}}$ is much smaller than $H_{\text {int }}$ and plays just the role of an instigator of the flavor conversion, generating initial perturbations. We hence drop it in the following analysis. This also implies that only the fast flavor conversion is considered. If the maximum wave number of vacuum oscillation $k_{\mathrm{vac}} \equiv \frac{1}{\hbar c} \frac{\Delta m_{\max }^{2}}{2 E}=\frac{10 \mathrm{MeV}}{E} \times 6.6 \times$ $10^{-6} \mathrm{~cm}^{-1}$ [45] becomes comparable to the growth rate $\sigma$ (discussed below) of the fast flavor conversion, however, $H_{\mathrm{vac}}$ should be reinstated and the slow mode also needs to be considered [29]. The collision term $\mathcal{C}[f]$ is also neglected in this study, since it is important not in the flavor conversion itself but in setting the background for it [31].

We work in the framework of two-flavor mixing. Then a small perturbation around the flavor eigenstate is expressed as

$$
f(x, \Gamma)=\left(\begin{array}{cc}
f_{v_{e}}(\Gamma) & 0 \\
0 & f_{\nu_{x}}(\Gamma)
\end{array}\right)+\frac{f_{c}(\Gamma)}{2}\left(\begin{array}{cc}
0 & S(x, \Gamma) \\
\bar{S}(x, \Gamma) & 0
\end{array}\right),
$$

where $f_{c}(\Gamma) \equiv f_{v_{e}}(\Gamma)-f_{v_{x}}(\Gamma)$ and the small off-diagonal component is denoted by $S$. Defining further the energy-integrated off-diagonal component $\mathscr{S}(x, v) \equiv$ $e^{i \Lambda_{c} \cdot x} \int_{-\infty}^{\infty} \frac{d E E^{2}}{2 \pi^{2}} S(x, \Gamma), \quad$ with $\quad \Lambda_{c}^{\mu} \equiv \sqrt{2} G_{F}\left[j_{e}^{\mu}-j_{x}^{\mu}+\right.$ $\left.\int d \Gamma f_{c}(\Gamma) v^{\mu}\right]$ and the angular intensity of ELN $\mathscr{G}(\boldsymbol{v}) \equiv \sqrt{2} G_{F} \int_{-\infty}^{\infty} \frac{d E E^{2}}{2 \pi^{2}} f_{c}(\Gamma)$, we can recast Eq. (3) for the off-diagonal component into

$$
v \cdot(i \partial) \mathscr{S}(x, \boldsymbol{v})+\int \frac{d^{2} \boldsymbol{v}^{\prime}}{4 \pi} \mathscr{G}\left(\boldsymbol{v}^{\prime}\right) v \cdot v^{\prime} \mathscr{S}\left(x, \boldsymbol{v}^{\prime}\right)=0
$$

to the linear order of $\mathscr{S}$. Note that the variation of $\Lambda$ is neglected, since we consider a patch of space much smaller than the background scale height and a period of time much shorter than the typical hydrodynamical timescale. For the plane wave ansatz $\mathscr{S}(x, \boldsymbol{v}) \equiv Q(\boldsymbol{v}) e^{i k \cdot x}$, a nontrivial solution of Eq. (5) exists if and only if

$$
\operatorname{det} \Pi(k)=0
$$

is satisfied for the polarization tensor given as

$$
\Pi^{\mu v}(k)=\eta^{\mu \nu}+\int \frac{d^{2} v}{4 \pi} \mathscr{G}(\boldsymbol{v}) \frac{v^{\mu} v^{v}}{v \cdot k} .
$$

The fast flavor conversion, which is regarded here as instability of the flavor eigenstate, occurs when the solution of Eq. (6), $k^{0}=\omega(\boldsymbol{k})$, has a positive imaginary part for some $\boldsymbol{k} \in \mathbb{R}^{3}$. Since analytic solutions are not expected in general, we need to solve Eq. (6) numerically, which is not an easy task [26,33,38-40].

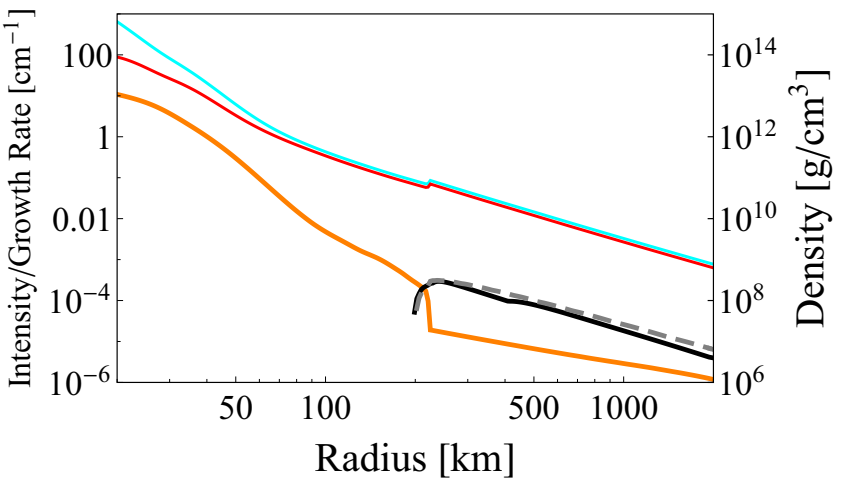

FIG. 1. Radial profiles of the baryonic mass density (orange solid line) and the number density of $v_{e}$ (cyan solid line) and $\bar{v}_{e}$ (red solid line) multiplied by $\sqrt{2} G_{F}(\hbar c)^{2}$. The black solid and gray dashed lines represent the growth rate of the fast flavor conversion for the standard-resolution $\left[\left(N_{E}, N_{\mu}\right)=(20,10)\right]$ and high-resolution $\left[\left(N_{E}, N_{\mu}\right)=(30,40)\right]$ simulations, respectively $\left(N_{E}\right.$ and $N_{\mu}$ denote the numbers of energy and angular grid points, respectively). The time is $100 \mathrm{~ms}$ after the bounce and the shock wave is located at $\sim 223 \mathrm{~km}$.

\section{REALISTIC MODELS}

Below we vindicate the above argument given for the bulb model by quantitatively analyzing the data obtained in our CCSN simulations with the full Boltzmann neutrino transport. Importantly, the ELN crossings in the preshock region are confirmed in all of our 1D and 2D models [46-49] and also in the 1D models of Tamborra et al., which are publicly available [50]. Tamborra et al. [18] reported that there was no ELN crossing in the latter models, which is not true however. ${ }^{2}$ In the following analysis, we employ the numerical data of a spherically symmetric $11.2 M_{\odot}$ CCSN model [47] as a representative case.

Figure 1 portrays the radial profiles of neutrino number densities and baryonic mass density as well as the approximate estimate of the growth rate of the fast flavor conversion given by the formula

$$
\sigma \sim \sqrt{-\left(\int_{\mathscr{G}(\boldsymbol{v})>0} \frac{d^{2} \boldsymbol{v}}{4 \pi} \mathscr{G}(\boldsymbol{v})\right)\left(\int_{\mathscr{G}(\boldsymbol{v})<0} \frac{d^{2} \boldsymbol{v}}{4 \pi} \mathscr{G}(\boldsymbol{v})\right)},
$$

which is motivated by the analysis of the two-beam model (see footnote 1) and not bad indeed as confirmed later by linear analysis. As shown in Fig. 1, the fast flavor conversion occurs at the preshock region and its growth rate is $\sim 10^{-4} \mathrm{~cm}^{-1}$. It should be stressed that the result is not an artifact of numerical diffusions in our CCSN simulations; indeed, the same simulation but with much higher resolutions yields essentially the same results (the gray dashed line in the same figure). ${ }^{3}$

\footnotetext{
${ }^{2}$ It seems that they overlooked or discarded this tiny crossing, which is almost invisible if the ELN distributions are plotted in linear scale.

${ }^{3}$ For this simulation and another with the coherent scattering turned off, only neutrino transport was recomputed for the fixed matter background. We confirmed the validity of this treatment, employing the standard setting.
} 


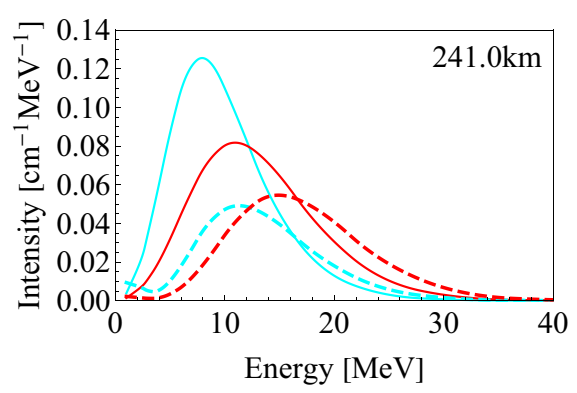

(a)

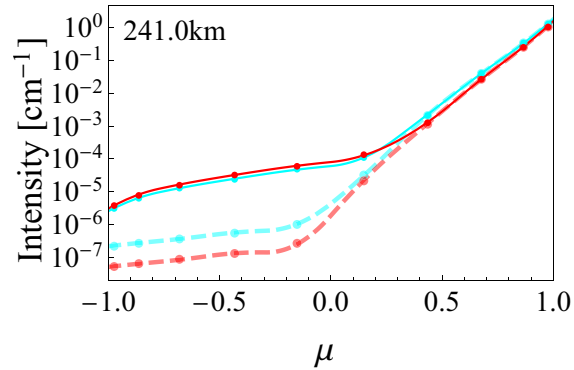

(b)

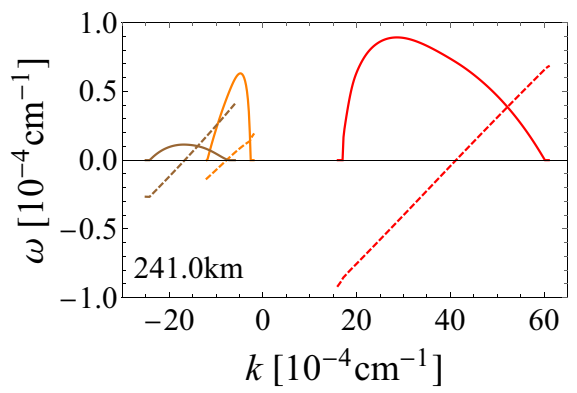

(c)

FIG. 2. (a) Energy spectra of $v_{e}$ (cyan) and $\bar{v}_{e}$ (red) at $r=241 \mathrm{~km}$. The solid and dashed lines are for $\mu=0.97$ (outgoing neutrinos) and $\mu=-0.87$ (ingoing neutrinos), respectively. A factor of $10^{5}$ is multiplied for the latter. (b) Angular distributions of neutrinos at the same radius with the same notation for colors. Dashed lines represent the results for the simulation without scatterings of heavy nuclei. (c) Complex $\omega$ as a function of real $k$ for unstable modes at $r=241 \mathrm{~km}$ derived by solving Eq. (6). The solid and dashed lines represent Im $\omega$ and $0.05 \operatorname{Re} \omega$, respectively. Note that there are three branches of the dispersion relation, which are distinguished by colors. The time is $100 \mathrm{~ms}$ after the bounce.

On the other hand, the fast flavor conversion is suppressed in the postshock region. It is attributed to the fact that almost all heavy nuclei are photodissociated in the postshock flows, which substantially reduces scattering opacities. In addition, the isotropic emission of $v_{e}$ via the electron capture by free protons is enhanced by shock heating and becomes the dominant weak process for inward-going neutrinos behind the shock wave. ${ }^{4}$ As a result, the tiny ELN crossing that could be induced by the scattering is eliminated and $v_{e}$ dominates over $\bar{v}_{e}$ in all directions.

We turn our attention to the detailed characteristics of the neutrino distributions in momentum space. For outward-going neutrinos, the average energy, which roughly corresponds to the energy at the peak of the spectrum, is higher for $\bar{v}_{e}$ than $v_{e}$, whereas the peak is higher for $v_{e}$ than $\bar{v}_{e}$ [see solid lines in Fig. 2(a)]; as a result, the number density of $v_{e}$ is slightly larger than that of $\bar{v}_{e}$, i.e., the ELN is positive [see also the solid lines at $\mu>0$ in Fig. 2(b)]. For inward-going neutrinos, on the other hand, both the peak of the spectrum and the average energy are higher for $\bar{v}_{e}$ than $v_{e}$ and hence $\bar{v}_{e}$ is more abundant than $v_{e}$ [see dashed lines in Fig. 2(a)], i.e., the ELN is negative. This indicates that the ELN crossing occurs, which is exactly what we predicted from our toy model. Indeed, it is confirmed that the neutrino angular distributions intersect at $\mu \sim 0.2$ as shown in Fig. 2(b).

To see more clearly the role of the scattering by heavy nuclei, we perform an additional simulation, in which we turn it off (see footnote 3 ). The angular distributions of neutrinos obtained in this simulation are displayed as dashed lines in Fig. 2(b). It is apparent that the outward-going neutrinos are almost intact whereas the inward-going neutrinos are strongly affected, and thus neutrinos are much less abundant and, more importantly, the ELN crossing disappears. We can hence conclude that the coherent scattering by heavy nuclei plays a crucial role in generating the ELN crossing.

\footnotetext{
${ }^{4}$ The latter claim may not always be true though. In fact, we find that the positron capture can dominate over the electron capture in some regions of the postshock flow in the presence of strong asymmetric neutrino emissions (see Ref. [49]).
}

Figure 2(c) displays the dispersion relation (DR) at $r=$ $241 \mathrm{~km}$ for $\boldsymbol{k}$ parallel to the radial direction, which gives the growth rate of the fast flavor conversion more precisely than Eq. (8). Note that the maximum growth rate derived from DR is $\sim 10^{-4} \mathrm{~cm}^{-1}$, which agrees roughly with the value estimated by Eq. (8), $2.94 \times 10^{-4} \mathrm{~cm}^{-1}$. More interestingly, the group velocity of these unstable modes $\left(v_{\mathrm{g}}=\right.$ $d \operatorname{Re} \omega / d k)$, which gives the growing direction of perturbations $[26,33,38-40]$, is $\sim 0.7 c$ and always positive, which implies that the flavor conversion proceeds in the outward direction.

Figure 3 shows the growth rates of the fast flavor conversion as a function of radius at different times. In the early postbounce phase (up to $\sim 30 \mathrm{~ms}$ after the bounce in this model), as is known well [51], the emission of $v_{e}$ dominates that of $\bar{v}_{e}$ and, as a result, $\epsilon$ is smaller than unity and the flavor conversion is suppressed, as expected from Eq. (2). Once $\bar{v}_{e}$ is produced substantially (at $\sim 50 \mathrm{~ms}$ ), it is confirmed that the

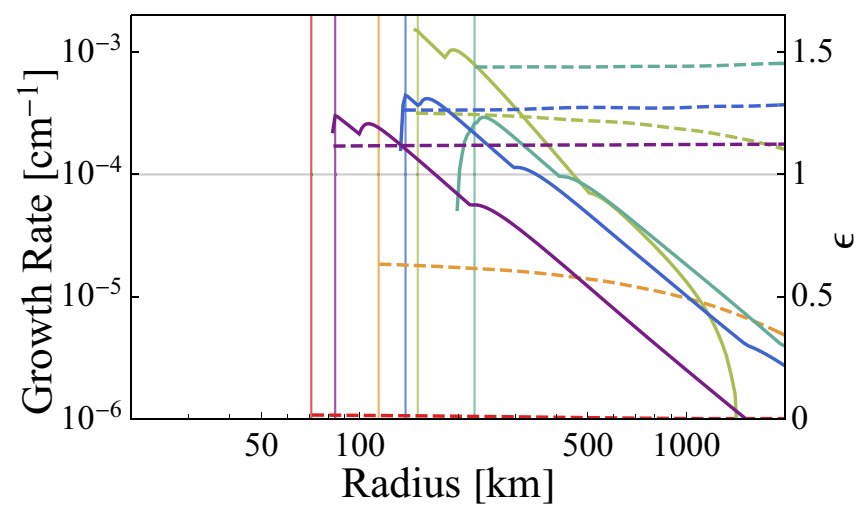

FIG. 3. Growth rates of the fast flavor conversion estimated by Eq. (8) (solid lines) and the ratio $\epsilon \equiv L_{\bar{v}_{e}} \bar{E}_{\bar{v}_{e}} / L_{v_{e}} \bar{E}_{v_{e}}$ outside the shock (dashed lines) as functions of the radius at different times: $10 \mathrm{~ms}$ (red), $30 \mathrm{~ms}$ (orange), $50 \mathrm{~ms}$ (lime), $100 \mathrm{~ms}$ (green), $200 \mathrm{~ms}$ (blue), and $400 \mathrm{~ms}$ (purple). The thin vertical lines indicate the shock positions at the same times. The lack of red and orange solid lines in this means no fast flavor conversion at the corresponding times. 
ELN crossing occurs in the preshock region and is sustained for the rest of the postbounce phase.

\section{CONCLUSION}

In this Rapid Communication we have presented the possibility of fast neutrino-flavor conversion in CCSNs. We have argued that it should be ubiquitous in the preshock region in the postbounce phase except for the very early period ( $\lesssim 30 \mathrm{~ms}$ after the bounce). The key ingredient is the coherent neutrino-nucleus scattering. We have demonstrated both analytically and numerically that the scattering induces the ELN crossing and then triggers the fast flavor conversion. We also found that the group velocities of unstable modes are always positive irrespective of their phase velocities, i.e., the fast flavor conversion may have an influence on the terrestrial observation of supernova neutrinos.

\section{ACKNOWLEDGMENTS}

We are grateful to S. Abbar for valuable discussions. T.M. was supported by JSPS Grant-in-Aid for JSPS Fellows (Grant No. 19J21244) from the Ministry of Education, Culture, Sports, Science and Technology (MEXT), Japan. H.N. was supported by Princeton University through DOE SciDAC4 Grant No. DE-SC0018297 (Award No. 00009650). This work was also supported by the Grantin-Aid for Scientific Research (Grants No. 15K05093, No. 25870099, No. 26104006, No. 16H03986, No. 17H06357, and No. 17H06365), HPCI Strategic Program of Japanese MEXT and K computer at the RIKEN (Projects No. hpci 160071, No. 160211, No. 170230, No. 170031, No. 170304 , No. hp180179, No. hp180111, and No. hp180239), and Waseda University Grant for Special Research Projects (Project No. 2018K-263).
[1] S. Hannestad, G. G. Raffelt, G. Sigl, and Y. Y. Y. Wong, Phys. Rev. D 74, 105010 (2006).

[2] H. Duan, G. M. Fuller, and Y. Z. Qian, Phys. Rev. D 74, 123004 (2006).

[3] G. G. Raffelt and A. Y. Smirnov, Phys. Rev. D 76, 081301(R) (2007).

[4] B. Dasgupta, A. Dighe, G. G. Raffelt, and A. Y. Smirnov, Phys. Rev. Lett. 103, 051105 (2009).

[5] A. Banerjee, A. Dighe, and G. Raffelt, Phys. Rev. D 84, 053013 (2011).

[6] S. Sarikas, D. de Sousa Seixas, and G. Raffelt, Phys. Rev. D 86, 125020 (2012).

[7] S. Sarikas, I. Tamborra, G. Raffelt, L. Hüdepohl, and H. T. Janka, Phys. Rev. D 85, 113007 (2012).

[8] J. F. Cherry, J. Carlson, A. Friedland, G. M. Fuller, and A. Vlasenko, Phys. Rev. Lett. 108, 261104 (2012).

[9] A. Mirizzi and P. D. Serpico, Phys. Rev. D 86, 085010 (2012).

[10] A. Mirizzi and P. D. Serpico, Phys. Rev. Lett. 108, 231102 (2012).

[11] G. Raffelt, S. Sarikas, and D. D. S. Seixas, Phys. Rev. Lett. 111, 091101 (2013).

[12] J. F. Cherry, J. Carlson, A. Friedland, G. M. Fuller, and A. Vlasenko, Phys. Rev. D 87, 085037 (2013).

[13] A. Mirizzi, Phys. Rev. D 88, 073004 (2013).

[14] S. Chakraborty and A. Mirizzi, Phys. Rev. D 90, 033004 (2014).

[15] S. Abbar and H. Duan, Phys. Lett. B 751, 43 (2015).

[16] B. Dasgupta and A. Mirizzi, Phys. Rev. D 92, 125030 (2015).

[17] Y. Yang and J. P. Kneller, Phys. Rev. D 96, 023009 (2017).

[18] I. Tamborra, L. Hüdepohl, G. G. Raffelt, and H.-T. Janka, Astrophys. J. 839, 132 (2017).

[19] V. Cirigliano, M. Paris, and S. Shalgar, J. Cosmol. Astropart. Phys. 11 (2018) 019.

[20] E. Rrapaj, arXiv:1905.13335.

[21] M. Zaizen, J. F. Cherry, T. Takiwaki, S. Horiuchi, K. Kotake, H. Umeda, and T. Yoshida, arXiv:1908.10594.

[22] S. Chakraborty, R. S. Hansen, I. Izaguirre, and G. G. Raffelt, J. Cosmol. Astropart. Phys. 03 (2016) 042.
[23] R. F. Sawyer, Phys. Rev. Lett. 116, 081101 (2016).

[24] B. Dasgupta, A. Mirizzi, and M. Sen, J. Cosmol. Astropart. Phys. 02 (2017) 019.

[25] I. Izaguirre, G. Raffelt, and I. Tamborra, Phys. Rev. Lett. 118, 021101 (2017).

[26] F. Capozzi, B. Dasgupta, E. Lisi, A. Marrone, and A. Mirizzi, Phys. Rev. D 96, 043016 (2017).

[27] B. Dasgupta and M. Sen, Phys. Rev. D 97, 023017 (2018).

[28] S. Abbar and H. Duan, Phys. Rev. D 98, 043014 (2018).

[29] S. Airen, F. Capozzi, S. Chakraborty, B. Dasgupta, G. Raffelt, and T. Stirner, J. Cosmol. Astropart. Phys. 12 (2018) 019.

[30] S. Abbar, H. Duan, K. Sumiyoshi, T. Takiwaki, and M. C. Volpe, Phys. Rev. D 100, 043004 (2019).

[31] F. Capozzi, B. Dasgupta, A. Mirizzi, M. Sen, and G. Sigl, Phys. Rev. Lett. 122, 091101 (2019).

[32] S. Abbar and M. C. Volpe, Phys. Lett. B 790, 545 (2019).

[33] C. Yi, L. Ma, J. D. Martin, and H. Duan, Phys. Rev. D 99, 063005 (2019).

[34] M. Delfan Azari, S. Yamada, T. Morinaga, W. Iwakami, H. Okawa, H. Nagakura, and K. Sumiyoshi, Phys. Rev. D 99, 103011 (2019).

[35] J. D. Martin, S. Abbar, and H. Duan, Phys. Rev. D 100, 023016 (2019).

[36] S. Shalgar and I. Tamborra, Astrophys. J. 883, 80 (2019).

[37] H. Nagakura, W. Iwakami, S. Furusawa, H. Okawa, A. Harada, K. Sumiyoshi, S. Yamada, H. Matsufuru, and A. Imakura, Astrophys. J. 854, 136 (2018).

[38] R. J. Briggs, Electron-Stream Interaction with Plasmas (MIT Press, Cambridge, 1964).

[39] E. M. Lifshitz and L. P. Pitaevskii, Physical Kinetics, Landau and Lifshitz: Course of Theoretical Physics Vol. 10 (Butterworth-Heinemann, Washington, DC, 1997), Chap. VI.

[40] T. Morinaga and S. Yamada, Phys. Rev. Research 2, 013045 (2020)

[41] M. T. Keil, G. G. Raffelt, and H.-T. Janka, Astrophys. J. 590, 971 (2002).

[42] I. Tamborra, B. Müller, L. Hüdepohl, H. T. Janka, and G. Raffelt, Phys. Rev. D 86, 125031 (2012). 
[43] See Supplemental Material at http://link.aps.org/supplemental/ 10.1103/PhysRevResearch.2.012046 for the derivations of Eqs. (1) and (2).

[44] S. W. Bruenn, Astrophys. J. Suppl. Ser. 58, 771 (1985).

[45] M. Tanabashi, K. Hagiwara, K. Hikasa, K. Nakamura, Y. Sumino, F. Takahashi, J. Tanaka, K. Agashe, G. Aielli, C. Amsler et al. (Particle Data Group), Phys. Rev. D 98, 030001 (2018).

[46] K. Sumiyoshi, S. Yamada, H. Suzuki, H. Shen, S. Chiba, and H. Toki, Astrophys. J. 629, 922 (2005).
[47] H. Nagakura, S. Furusawa, H. Togashi, S. Richers, K. Sumiyoshi, and S. Yamada, Astrophys. J. Suppl. 240, 38 (2019).

[48] H. Nagakura, K. Sumiyoshi, and S. Yamada, Astrophys. J. 880, L28 (2019).

[49] H. Nagakura, T. Morinaga, C. Kato, and S. Yamada, Astrophys. J. 886, 139 (2019).

[50] Garching Core-Collapse Supernova Archive, https://wwwmpa. mpa-garching.mpg.de/ccsnarchive/data/Tamborra2017/ (Max Planck Institute for Astrophysics, Garching, 2017).

[51] B. Müller, Annu. Rev. Nucl. Part. Sci. 69, 253 (2019). 